\title{
1 Viral infection enhances vomocytosis of intracellular fungi via Type I
}

\section{2 interferons}

3 Paula I Seoane, Leanne M. Taylor-Smith ${ }^{1}$, David Stirling', Lucy C. K. Bell ${ }^{2}$, Mahdad

$4 \quad$ Noursadeghi ${ }^{2}$, Dalan Bailey ${ }^{3}$, Robin C. May ${ }^{1 *}$

5

6 Institute of Microbiology \& Infection and School of Biosciences, University of Birmingham,

7 Edgbaston, Birmingham, B15 2TT, UK

$8{ }^{2}$ Division of Infection and Immunity, University College London, Gower Street, London WC1E

$96 B T, U K$

${ }^{3}$ The Pirbright Institute, Ash Rd, Surrey, GU24 ONF, UK

*Correspondence to: r.c.may@bham.ac.uk

14 Abstract

15 Cryptococcus neoformans is an opportunistic human pathogen, which causes serious disease in immunocompromised hosts. Infection with this pathogen is particularly relevant in $\mathrm{HIV}^{+}$

17 patients, where it leads to around 200,000 deaths per annum. A key feature of cryptococcal pathogenesis is the ability of the fungus to survive and replicate within the phagosome of macrophages, as well as its ability to escape via a novel non-lytic mechanism known as vomocytosis. We have been exploring whether viral infection affects the interaction between C. neoformans and macrophages. Here we show that viral infection enhances cryptococcal vomocytosis without altering phagocytosis or intracellular proliferation of the fungus. This effect occurs with distinct, unrelated human viral pathogens and is recapitulated when macrophages are stimulated with the anti-viral cytokine interferon alpha (IFN $\alpha)$. Importantly, the effect is abrogated when type-I interferon signalling is blocked, thus underscoring the 
importance of type-I interferons in this phenomenon. Our results highlight the importance of

27 incorporating specific context cues while studying host-pathogen interactions. By doing so, we

28 found that acute viral infection may trigger the release of latent cryptococci from intracellular

29 compartments, with significant consequences for disease progression.

31 Non-Technical Author Summary

32 Infectious diseases are typically studied in the laboratory in isolation, but in real life people 33 often encounter multiple infections simultaneously. Here we investigate how the innate

34 immune response to the fatal fungus Cryptococcus neoformans is influenced by viral 35 coinfection. Whilst virally-infected macrophages retain a normal capacity to engulf and kill 36 Cryptococci, they demonstrate a dramatically enhanced propensity to expel them via the 37 process known as non-lytic expulsion or vomocytosis. Activation of vomocytosis is 38 independent of the type of virus encountered, since both HIV and measles (two entirely 39 unrelated viral pathogens) trigger the same effect. Instead it is driven by interferon- $\alpha$, a generic 40 'antiviral' response, which signals back to the infected macrophage, triggering expulsion of the 41 fungus. We propose that this hitherto unobserved phenomenon represents a 'reprioritisation' 42 pathway for innate immune cells, by which they can alter the frequency with which they expel one pathogen (Cryptococcus) depending on the level of threat from a secondary viral infection. 
47 Since their discovery in 1957 by Isaacs and Lindenmann (1), the antiviral effects of type I

\section{Introduction}

interferons have been well documented (2-4). More recently, their roles in non-viral infections

have been investigated $(5,6)$. Different bacterial stimuli have been shown to elicit type I interferon production, and in turn these so called "antiviral cytokines" play a role in the outcome of bacterial infections (7-9). This stems in part from the complex and sometimes contradictory effects that type I interferons have on host cells, for instance in enhancing inflammatory responses in some infectious settings (6) to preventing hyperinflammation in others $(10,11)$, and even affecting the priming of immune responses at lymph nodes (12).

To date, little is known about the interplay between type I interferons and fungal infections, despite the fact that many life-threatening fungal infections occur in the context of chronic viral infection. This is particularly true of Cryptococcus neoformans, a globally distributed opportunistic pathogen that is responsible for nearly 200,000 deaths per year in human immunodeficiency virus (HIV) infected people, where it causes cryptococcal meningitis (13). Extensive work over many years has demonstrated that a key feature of cryptococcal pathogenesis is the ability of the fungus to survive, proliferate within, and then escape from, host macrophages (14-17). Macrophages are among the first immune cells to encounter the fungus within the human host (18), and thus are very important in the fight against this pathogen. These cells are able to phagocytose and contain the threat, as happens in immunocompetent hosts, but can also by hijacked by Cryptococcal cells and used as a "Trojan horse" to disseminate to distal sites within the body, particularly to the central nervous system (19). Engulfed Cryptococcal cells can escape from host macrophages through lytic or non-lytic mechanisms, the latter being known as vomocytosis or non-lytic extrusion $(20,21)$. Most 
70 studies to date have focused on the interaction of Cryptococcus with healthy host cells, and

71 consequently how this intracellular lifestyle may be impacted by viral coinfection remains

72 unknown.

73

74 Here we show that viral infections enhance vomocytosis of Cryptococci from infected

75 macrophages, without affecting phagocytosis or intracellular proliferation rate of the fungus.

76 This effect is lost when signalling through the type I interferon receptor is blocked, and can be

77 recapitulated by addition of exogenous IFNa. Thus, antiviral responses by the host have a

78 hitherto unexpected impact on the release of intracellular pathogens by vomocytosis. 
Materials and Methods

80

All reagents were purchased from SIGMA unless otherwise stated.

81

\section{Cryptococcus Strains}

Cryptococcal strains were grown in Yeast Peptone Dextrose (YPD) broth (2\% glucose, 1\% peptone and $1 \%$ yeast extract) at $25^{\circ} \mathrm{C}$ on a rotator $(20 \mathrm{rpm})$. Yeast from overnight cultures were centrifuged at $6500 \mathrm{rpm}$ for 2 minutes and resuspended in PBS at the required concentration. All experiments were carried out using C. neoformans var. grubii serotype A strain Kn99 $\alpha$. Wildtype, GFP- (22) or mCherry-expressing (23) derivatives of Kn99 $\alpha$ were used, as stated for each figure.

\section{Virus strains}

HIV-1 virus stocks were generated by transfection of human embryonic kidney $293 \mathrm{~T}$ cells (European Collection of Authenticated Cell Cultures) as previously described $(24,25)$. The R9HIV $\Delta e n v$ virus was derived from clade B HIV-1 strain (NL43) with 500bp deletion in env, pseudotyped with vesiculostomatitis virus $G$ envelope. SIV3mac single round virus like particles (VLPs) containing vpx (SIV3vpx) were generated by transfection into 293T cells with pSIV3+ and pMDG plasmids $(26,27)$. At 48, $72 \mathrm{~h}$ and $96 \mathrm{~h}$ viral containing supernatant was harvested, centrifuged at $800 \mathrm{x}$ g for $10 \mathrm{~min}$ and filtered through $0.45 \mathrm{um}$ filter then centrifuged on a $20 \%$ sucrose cushion at $20,000 \mathrm{x}$ g for $2 \mathrm{~h}$ at $4{ }^{\circ} \mathrm{C}$. Purified virus was then re-suspended in RPMI media and frozen at $-80^{\circ} \mathrm{C}$. To quantify single round $\mathrm{HIV}$ infection, a vial was thawed for each harvest and serial dilutions used to infect CCR5/CD4 and CXCR4/CD4 transfected NP-2 cells. At $72 \mathrm{~h}$ post infection wells were fixed in ice cold acetone-methanol and infected cells were identified by staining for $\mathrm{p} 24$ protein using a 1:1 mixture of the anti-p24 monoclonal antibodies EVA365 and EVA366 (NIBSC, Center for AIDS Reagents, UK). Infected cells 
were detected by light microscopy to provide a virus titre (focus-forming $\mathrm{U} / \mathrm{mL}$ ). The SIV3vpx particles were quantified after thawing using a reverse transcriptase (RT) assay colorimetric kit (Roche) following the manufacturer's instructions to provide a RT ng/mL titre.

Recombinant $\mathrm{MeV}$ strain IC323 expressing green fluorescent protein (MeV-GFP) was generated as previously reported by Hashimoto et al. (28) MeV-GFP represents a virulent field isolate from Japan (Ichinose-B (IC-B) strain) and was isolated from a patient with acute measles in 1984 (29). For the generation of virus stocks, Vero (ATCC CCL-81) cells overexpressing human SLAMF1 receptor (vero-hSLAM cells) were grown in T75 tissue culture flasks to approximately $80 \%$ confluency in DMEM supplemented with $0.4 \mathrm{mg} / \mathrm{mL}$ G418. Flasks were infected with MeV-GFP at an MOI of 0.01:1 in $5 \mathrm{~mL}$ media for 1 hour at $37^{\circ} \mathrm{C}$. After $1 \mathrm{~h}$ a further $10 \mathrm{~mL}$ of DMEM supplemented with $10 \% \mathrm{FBS}$ was added and infection allowed to continue for $48 \mathrm{~h}$. At harvest the flasks were frozen to $-80^{\circ} \mathrm{C}$. After thawing, the collected supernatants were centrifuged at $2500 \mathrm{rpm}$ for $10 \mathrm{~min}$ at $4{ }^{\circ} \mathrm{C}$ to pellet cell debris. Aliquoted virus in supernatant was then frozen to $-80^{\circ} \mathrm{C}$. MeV-GFP viruses were then titred using the TCID-50 method. Vero-hSLAM cells were seeded into flat-bottomed 96 well plates and infected with serial dilutions of thawed MeV-GFP in triplicate. After $72 \mathrm{~h}$, wells were scored for positive or negative infection under UV illumination on a Nikon TE2000 microscope.

\section{Ethics Statement}

All work with human tissue was approved by the University of Birmingham Ethics Committee under reference ERN_10-0660. Samples were collected specifically for this work and were not stored beyond the duration of the experiments described herein. All donors provided written consent prior to donation. 


\section{Human macrophage isolation and culture}

$13120-40 \mathrm{~mL}$ of blood were drawn from healthy donors by venepuncture. $6 \mathrm{~mL}$ of whole blood

132 were carefully layered on top of a double layer of Percoll (densities of 1.079 and $1.098 \mathrm{~g} / \mathrm{mL}$ ).

133 Samples were centrifuged in a swing bucket rotor at $150 \mathrm{~g}$ for 8 minutes, followed by 10 minutes at $1200 \mathrm{~g}$, with acceleration and break set to zero. The resulting white disc of peripheral blood mononuclear cells (PBMC) was transferred to a clean vial and incubated with red blood cell lysis buffer at a ratio of 1:3 for 3 minutes, with gentle mixing throughout to prevent clot formation. Cells were then washed with ice cold PBS twice, with centrifugation at $400 \mathrm{~g}$ for 6 minutes in between each wash, and counted with a haemocytometer. $1 \times 10^{6} \mathrm{PBMC}$ were seeded onto 48-well plates in RPMI-1640 media containing 1\% penicillin/streptomycin, 5\% heatinactivated AB human serum and $20 \mathrm{ng} / \mathrm{mL}$ M-CSF (Invitrogen). Cells were washed with PBS and resuspended in fresh media on days 3 and 6 of differentiation. Macrophages were ready to use on day 7. A yield of $1 \times 10^{5}$ macrophages per well was estimated.

\section{Cryptococcus infection}

145 Fungi were opsonised with $10 \%$ human $\mathrm{AB}$ serum or $18 \mathrm{~B} 7$ antibody (a kind gift from Arturo

146 Casadevall) for 1 hour and then added to macrophages at a multiplicity of infection of 10:1.

147 Infection was carried out in serum free-media, at $37^{\circ} \mathrm{C}$ with $5 \% \mathrm{CO}_{2}$. After 2 hours, cells were 148 washed 3 times with PBS to remove any extracellular fungi and fresh serum free-media was 149 added.

\section{Drug treatments}

152 Exogenous compounds were added to macrophages at two stages; when infecting with

153 Cryptococcus and again when replenishing with fresh media after removing extracellular fungi. 
154 Compounds tested include interferon alpha (IFN $\alpha$ ) at concentrations ranging from 5 to 100

155

156

157

158

159

160

161

162

163

164

165

166

167

168

169

170

171

172

173

174

175 Live imaging

176 Infected samples were kept at $37^{\circ} \mathrm{C}$ with $5 \% \mathrm{CO}_{2}$ in the imaging chamber of a Ti-E Nikon

177 Epifluorescence microscope. Images were taken every 5 minutes over an 18-hour period and

$\mathrm{pg} / \mathrm{mL}$ (Bio-Techne), polyinosinic-polycytidilic acid (polyIC) at 3 and $30 \mathrm{ng} / \mathrm{mL}$ (Invivogen), type-I interferon receptor inhibitor (IFNARinh) at $2.5 \mu \mathrm{g} / \mathrm{mL}$ (pbl assay science).

\section{Co-infection assay}

Human monocyte-derived macrophages were infected with either attenuated human immunodeficiency virus (HIV) or MeV-GFP as follows:

For attenuated HIV co-infections, 24h before cryptococcal infection, human monocyte-derived macrophages were infected either with R9HIV $\Delta e n v$ at a MOI of 10:1, SIV3vpx at $3 \mathrm{ng} / \mathrm{mL}$ or both in serum free RPMI. At $24 \mathrm{~h}$ post infection duplicate wells were fixed in ice cold acetonemethanol and infected cells were identified by staining for p24 protein as described above. Experimental wells were infected with antibody opsonised-Cryptococcus Kn99 $\alpha$-GFP for 2 hours, washed to remove extracellular fungal cells, and replenished with fresh serum freemedia.

Alternatively, macrophages were infected with MeV-GFP at an MOI of 5:1 in serum freemedia and kept at $37^{\circ} \mathrm{C}$ with $5 \% \mathrm{CO}_{2}$. After 24 hours, cells were washed with PBS and fresh media, supplemented with 5\% heat-inactivated human AB serum, was added. After 3 days, cells were co-infected with serum opsonised-Cryptococcus Kn99 $\alpha$-mCherry for 2 hours, washed to remove extracellular fungal cells, and replenished with fresh serum free-media.

\footnotetext{
compiled into a single movie file using NIS Elements software. Movies were blinded by a third
} 
179 party before manual scoring for phagocytosis of Cryptococcus, virus infection rates, vomocytosis events, intracellular proliferation rates and macrophage integrity.

\section{Growth curve assay}

183 A 10-fold diluted cryptococcal overnight culture was inoculated into YPD broth in a 48-well plate (final dilution in well: 1000-fold), in the presence or absence of type-I interferons. The plate was sealed with a breathable membrane and incubated at $37^{\circ} \mathrm{C}$ within a fully automated plate reader (FLUOStar, BMG Omega). Optical density readings at $600 \mathrm{~nm}$ were taken every 30 minutes over a 24 hour-period, with orbital shaking in between readings.

\section{Data analysis}

190 Statistical analysis was performed using GraphPad Prism 6. Categorical data of phagocytosis

191 or vomocytosis occurrence in the different conditions was assessed using $\mathrm{Chi}^{2}$ test and Fisher's 192 exact test. If data was normally distributed as assessed by Shapiro-Wilk test, then it was 193 compared using Student's t test. Figures show percentage of cryptococcus-infected macrophages experiencing at least one vomocytosis event within each experiment. For intracellular proliferation rates, data was analysed using Mann-Whitney test. Growth curves were fitted to sigmoidal curves and the parameters were compared using Kruskal-Wallis test. All data shown corresponds to at least three independent experiments.

198 Raw data (collated manual counts for multiple timelapse movies) are provided as supplemental 199 material for each figure. Original timelapse movies, upon which manual scoring was performed, are freely available upon request from the authors. 


\section{Results}

203 Given the relevance of cryptococcosis to $\mathrm{HIV}^{+}$patients (13), we set out to test whether HIV

204 infection had an effect on vomocytosis of C. neoformans. Human monocyte-derived macrophages were infected with HIV-1 capable of a single-round of infection and subsequently with $C$. neoformans and then used for time-lapse imaging over 18 hours. Subsequent scoring

207 showed that virally infected cells had a significantly higher occurrence of cryptococcal vomocytosis (Figure 1A), whilst fungal uptake and intracellular proliferation were unaltered (Figure 1C, 1E).

210

211 The experimental HIV system we used here includes co-transduction with SIV3vpx VLPs in

212 order to counteract the antiviral effect of SAMHD1 and ensure maximal HIV infection of the macrophages $(26,30)$ (Figure S1A). Interestingly, we noted that the addition of SIV3vpx or R9HIV $\Delta e n v$ alone also increased vomocytosis (Figure S1B). Since neither condition results in widespread viral infection of host cells, this suggested that the enhancement of vomocytosis occurs at the level of viral detection, rather than being a consequence of active HIV infection.

To explore this further, we tested whether vomocytosis was altered in macrophages infected with an unrelated macrophage-tropic virus (31); measles (MeV, Figure 1B). The measles strain used represents a virulent field isolate from Japan. Once again, infection with the virus resulted in significantly enhanced vomocytosis of Cryptococcus. Neither HIV nor measles infection affected uptake of Cryptococcus nor the intracellular proliferation rate (IPR) of the fungus rather than fungal pathogenicity per se, and that it is independent of the type of virus. 
226 To test whether active viral infection was required for enhanced vomocytosis, we mimicked

227 the effect of viral exposure by stimulating macrophages with polyinosinic-polycytidilic acid

228 (polyIC). PolyIC is a double-stranded RNA synthetic analogue, which is known to trigger

229 antiviral responses by binding to TLR3 (32). Human monocyte-derived macrophages were

230 stimulated with polyIC and infected with $C$. neoformans simultaneously. Infected cells were imaged over 18 hours and scored for vomocytosis (Figure 2A). As with HIV or MeV infection, polyIC stimulation enhanced vomocytosis of Cryptococcus. Thus, it is likely that the antiviral reaction of the host macrophage, rather than an aspect of viral pathogenesis, is the trigger for enhanced vomocytosis from infected host cells.

The hallmark of the cellular anti-viral response is the induction of type-I interferons. Among these, the best studied are IFN $\alpha$ and IFN $\beta$. During HIV infection specifically, the induction of IFN $\alpha$ is the most relevant (33). We therefore tested whether the impact of viral infection on vomocytosis could be recapitulated by exposure to interferon- $\alpha$ (IFN $\alpha$ ). Stimulation of human monocyte-derived macrophages with $10 \mathrm{pg} / \mathrm{mL}$ IFN $\alpha$ (a level that closely matches that seen in HIV-infected patients (33)) resulted in significantly enhanced vomocytosis of Cryptococcus

242 (Figure 2B) without altering cryptococcal growth, uptake or IPR (Figure S2). Interestingly, we noticed that higher doses of IFN $\alpha$ suppressed this effect, suggesting that the impact of

244 interferons on vomocytosis can be rapidly saturated.

246 To confirm that type-I interferons were behind the increase in vomocytosis observed, we 247 performed the viral infection experiments in the presence of a type-I interferon receptor 248 (IFNAR) inhibitor (Figure 3). The addition of IFNAR inhibitor blocked the enhancement of 249 vomocytosis otherwise elicited by viral infection in both HIV- and Measles-infection settings, confirming that type-I interferon signalling is necessary for this effect. Interestingly, this effect 
251 was particularly prominent on virally infected cells rather than neighbouring cells which were

252 not infected (Non-MeV; Figure 3B), suggesting that the impact of IFN

253 vomocytosis is highly localised and specific to the autocrine responses occurring within

254 infected cells, rather than endocrine responses mediated through cytokines. 


\section{Discussion}

257 In this study we set out to explore the consequences, if any, of viral infection on Cryptococcal

258 infection, focusing on the non-lytic escape mechanism known as vomocytosis. Infection with

259 either HIV or measles virus led to an enhancement in vomocytosis of C. neoformans, without

260 affecting uptake or intracellular proliferation of the fungus (Figure 1), an effect that could be recapitulated by stimulation with IFN $\alpha$ and abrogated when signalling from type-I interferon receptor was blocked (Figures 2 and 3). Thus, viral coinfection stimulates expulsion of intracellular fungi via Type I interferon signalling.

The effect was seen using two distinct viral pathogens which differ, among other parameters, in the magnitude of anti-viral response they elicit in human macrophages. Relative to other

Nonetheless, the low levels of type-I interferons induced by HIV, potentially enhanced by the co-infection with Cryptococcus, are sufficient to have a significant effect on vomocytosis. Infection with measles virus has been reported to induce limited production of type-I interferons in macaque models, albeit with potent induction of interferon-stimulated genes (34, 35). To date, there is no direct correlation between measles infection and cryptococcosis. However, given that both pathogens have a distinct respiratory phase it is possible that they interact within this shared niche, potentially through low doses of antiviral signalling.

Why might antiviral signalling induce vomocytosis? One possibility is that vomocytosis serves to "reset" phagocytes that have been unable to kill their prey, thus allowing them to serve a useful purpose in phagocytosing other pathogens rather than remaining "unavailable". In that 
localised infection, returning macrophages to functionality faster than would otherwise occur. others it may serve to inadvertently disseminate the fungus to distal sites.

285 This is supported by previous reports showing variable outcomes of interferon signalling on cryptococcal infection in mice. Sato et al. (36) showed that IFNARKO mice have lower fungal for the host during cryptococcal infection. Supporting this notion but using the sister species C. gatti, Oliveira et al (37) show that infection with influenza virus worsens the prognosis of subsequent fungal infection. On the other hand, Sionov et al (38) showed that stimulation with either C. neoformans or C. gatti infection. This effect was time-dependent, with the protective effect of pICLC treatment only occurring if administered during the first 72 hpi before the fungus reaches the brain. A tempting model, therefore, is that stimulating vomocytosis via accelerate disease progression.

Taken together, our findings therefore suggest that the antiviral response, and IFN $\alpha$ in particular, induce the expulsion of intracellular cryptococci and that this effect could be

301 advantageous or detrimental to the host, depending on the localization of the infected 302 phagocyte and timing of the event. 
305 PIS, LMTS and RCM are supported by funding from the European Research Council under

306 the European Union's Seventh Framework Programme (FP/2007-2013)/ERC Grant

307 Agreement No. 614562. RCM holds a Wolfson Royal Society Research Merit Award and PIS

308 is supported by a Darwin Trust scholarship. MN is supported by a Wellcome Trust Investigator

309 Award (207511/Z/17/Z) and the NIHR Biomedical Research Centre at University College

310 London Hospitals.

311 


\section{References}

313

314 1. Isaacs A, Lindenmann J. Virus interference. I. The interferon. Proc R Soc Lond B Biol Sci. 1957;147(927):258-67.

316 2. Haller O, Arnheiter H, Gresser I, Lindenmann J. Virus-specific interferon action.

317 Protection of newborn Mx carriers against lethal infection with influenza virus. J Exp Med.

318 1981;154(1):199-203.

319 3. Muller U, Steinhoff U, Reis LF, Hemmi S, Pavlovic J, Zinkernagel RM, et al.

320 Functional role of type I and type II interferons in antiviral defense. Science. 1994;264(5167):1918-21.

322 4. Yan N, Chen ZJ. Intrinsic antiviral immunity. Nat Immunol. 2012;13(3):214-22.

323 5. MacMicking JD. Interferon-inducible effector mechanisms in cell-autonomous immunity. Nat Rev Immunol. 2012;12(5):367-82.

325 6. Mancuso G, Midiri A, Biondo C, Beninati C, Zummo S, Galbo R, et al. Type I IFN 326 signaling is crucial for host resistance against different species of pathogenic bacteria. $\mathrm{J}$ 327 Immunol. 2007;178(5):3126-33.

328 7. Bergstrom B, Aune MH, Awuh JA, Kojen JF, Blix KJ, Ryan L, et al. TLR8 Senses

329 Staphylococcus aureus RNA in Human Primary Monocytes and Macrophages and Induces 330 IFN-beta Production via a TAK1-IKKbeta-IRF5 Signaling Pathway. J Immunol. $3312015 ; 195(3): 1100-11$.

332 8. Kagan JC, Su T, Horng T, Chow A, Akira S, Medzhitov R. TRAM couples endocytosis 333 of Toll-like receptor 4 to the induction of interferon-beta. Nat Immunol. 2008;9(4):361-8.

334 9. Mancuso G, Gambuzza M, Midiri A, Biondo C, Papasergi S, Akira S, et al. Bacterial 335 recognition by TLR7 in the lysosomes of conventional dendritic cells. Nat Immunol. 2009;10(6):587-94. 

production induced by Streptococcus pyogenes-derived nucleic acids is required for host protection. PLoS Pathog. 2011;7(5):e1001345. 2017;36(16):2404-18.

346 13. Rajasingham R, Smith RM, Park BJ, Jarvis JN, Govender NP, Chiller TM, et al. Global

347 burden of disease of HIV-associated cryptococcal meningitis: an updated analysis. Lancet Infect Dis. 2017;17(8):873-81.

14. Alvarez M, Casadevall A. Cell-to-cell spread and massive vacuole formation after

Cryptococcus neoformans infection of murine macrophages. BMC Immunol. 2007;8:16.

351 15. Feldmesser M, Kress Y, Novikoff P, Casadevall A. Cryptococcus neoformans is a

352 facultative intracellular pathogen in murine pulmonary infection. Infect Immun. 2000;68(7):4225-37.

354 16. Tucker SC, Casadevall A. Replication of Cryptococcus neoformans in macrophages is accompanied by phagosomal permeabilization and accumulation of vesicles containing 356 polysaccharide in the cytoplasm. Proc Natl Acad Sci U S A. 2002;99(5):3165-70.

357 17. Voelz K, May RC. Cryptococcal interactions with the host immune system. Eukaryot 358 Cell. 2010;9(6):835-46.

359 18. Osterholzer JJ, Milam JE, Chen GH, Toews GB, Huffnagle GB, Olszewski MA. Role 360 of dendritic cells and alveolar macrophages in regulating early host defense against pulmonary 361 infection with Cryptococcus neoformans. Infect Immun. 2009;77(9):3749-58. 
362

363

364

19. Sorrell TC, Juillard PG, Djordjevic JT, Kaufman-Francis K, Dietmann A, Milonig A, et al. Cryptococcal transmigration across a model brain blood-barrier: evidence of the Trojan horse mechanism and differences between Cryptococcus neoformans var. grubii strain H99 and Cryptococcus gattii strain R265. Microbes Infect. 2016;18(1):57-67.

20. Alvarez M, Casadevall A. Phagosome extrusion and host-cell survival after Cryptococcus neoformans phagocytosis by macrophages. Curr Biol. 2006;16(21):2161-5.

21. Ma H, Croudace JE, Lammas DA, May RC. Expulsion of live pathogenic yeast by macrophages. Curr Biol. 2006;16(21):2156-60.

22. Garelnabi M, Taylor-Smith LM, Bielska E, Hall RA, Stones D, May RC. Quantifying donor-to-donor variation in macrophage responses to the human fungal pathogen Cryptococcus neoformans. PLoS One. 2018;13(3):e0194615.

23. Upadhya R, Lam WC, Maybruck BT, Donlin MJ, Chang AL, Kayode S, et al. A fluorogenic C. neoformans reporter strain with a robust expression of m-cherry expressed from a safe haven site in the genome. Fungal Genet Biol. 2017;108:13-25.

24. Rasaiyaah J, Tan CP, Fletcher AJ, Price AJ, Blondeau C, Hilditch L, et al. HIV-1 evades innate immune recognition through specific cofactor recruitment. Nature. 2013;503(7476):402-5.

25. Tsang J, Chain BM, Miller RF, Webb BL, Barclay W, Towers GJ, et al. HIV-1 infection of macrophages is dependent on evasion of innate immune cellular activation. AIDS. 2009;23(17):2255-63.

26. Goujon C, Arfi V, Pertel T, Luban J, Lienard J, Rigal D, et al. Characterization of simian immunodeficiency virus SIVSM/human immunodeficiency virus type 2 Vpx function in human myeloid cells. J Virol. 2008;82(24):12335-45. 
(CD150)-independent measles virus entry as revealed by recombinant virus expressing green complementation of 'non-macrophage tropic' R5 viruses reveals robust entry of infectious HIV1 cores into macrophages. Retrovirology. 2014;11:25. 2018;3(3).

32. Alexopoulou L, Holt AC, Medzhitov R, Flavell RA. Recognition of double-stranded 400 RNA and activation of NF-kappaB by Toll-like receptor 3. Nature. 2001;413(6857):732-8. disease markers. PLoS One. 2013;8(2):e56527.

404 34. Shivakoti R, Siwek M, Hauer D, Schultz KL, Griffin DE. Induction of dendritic cell 405 production of type I and type III interferons by wild-type and vaccine strains of measles virus: role of defective interfering RNAs. J Virol. 2013;87(14):7816-27.

407 35. Shivakoti R, Hauer D, Adams RJ, Lin WH, Duprex WP, de Swart RL, et al. Limited in wild-type strains of measles virus. J Interferon Cytokine Res. 2015;35(4):292-301. 
410 36. Sato K, Yamamoto H, Nomura T, Matsumoto I, Miyasaka T, Zong T, et al.

411 Cryptococcus neoformans Infection in Mice Lacking Type I Interferon Signaling Leads to

412 Increased Fungal Clearance and IL-4-Dependent Mucin Production in the Lungs. PLoS One.

$413 \quad 2015 ; 10(9): \mathrm{e} 0138291$.

414 37. Oliveira LVN, Costa MC, Magalhaes TFF, Bastos RW, Santos PC, Carneiro HCS, et

415 al. Influenza A Virus as a Predisposing Factor for Cryptococcosis. Front Cell Infect Microbiol.

$416 \quad 2017 ; 7: 419$.

417 38. Sionov E, Mayer-Barber KD, Chang YC, Kauffman KD, Eckhaus MA, Salazar AM, et

418 al. Type I IFN Induction via Poly-ICLC Protects Mice against Cryptococcosis. PLoS Pathog. $419 \quad 2015 ; 11(8): \mathrm{e} 1005040$.

420

421 


\section{Figure Captions}

423 Fig 1. Viral infection enhances vomocytosis of $C$. neoformans

424 Human monocyte-derived macrophages were infected with HIV (left) or measles virus (right)

425 and subsequently infected with C. neoformans. Time-lapse microscopy videos were manually

426 scored for vomocytosis (top), uptake (middle) and intracellular proliferation rate of $C$.

427 neoformans (bottom). A-B Graphs show percentage of cryptococcus-infected macrophages

428 which have experienced at least one vomocytosis event. C-D Percentage of crytptococus-

429 infected macrophages. E-F Intracellular proliferation rate of C. neoformans over 18 hours. In

430 all cases, data corresponds to at least 3 independent experiments. Categorical vomocytosis and

431 phagocytosis data was analysed by $\mathrm{Chi}^{2}$ test followed by Fisher's exact test. $* \mathrm{p}<0.05 ; * * * * \mathrm{p}$

$432<0.0001$. IPR data was analysed using Mann-Whitney test.

433

434

Fig 2. Antiviral response increases vomocytosis

435 Human monocyte-derived macrophages were stimulated with different doses of polyIC (A) or

436 IFNa (B), and infected with C. neoformans. Graphs show Mean + SD of percentage of

437 cryptococcus-infected macrophages which have experienced at least one vomocytosis event.

$438 \mathrm{Chi}^{2}$ test followed by Fisher's exact test performed on raw vomocytosis counts. Data 439 corresponds to at least three independent experiments.

440

441 Fig 3. Type-I interferon signalling is necessary to enhance vomocytosis

442 Human monocyte-derived macrophages were infected with HIV (A) or GFP-expressing

443 measles virus (MeV-GFP, B) and subsequently with mCherry-expressing C. neoformans

444 (Kn99 $\alpha$-mCherry), in the presence or absence of an IFNAR blocking antibody. GFP negative

445 cells, which did not have an active Measles infection, were termed "Non-MeV". Graph shows

446 Mean + SD of percentage of Cryptococcus-infected macrophages which have experienced at 
447 least one vomocytosis event. Fisher's exact test performed on raw vomocytosis counts. Data

448 corresponds to two and three biological repeats, respectively.

449

450 Supporting information

\section{$451 \quad$ Fig S1}

452 A. Human monocyte-derived macrophages were infected with VLPs as indicated. After 24

453 hours, viral infection was assessed by p24 staining (blue).

454 B. Cells were infected with VLPs as indicated, and subsequently infected with C. neoformans.

455 Time-lapse microscopy videos were manually scored for vomocytosis. Graph shows

456 percentage of cryptococcus-infected macrophages which have experienced at least one

457 vomocytosis event. $\mathrm{Chi}^{2}$ test followed by Fisher's exact test performed on raw vomocytosis

458 counts from 5 independent experiments.

459

$460 \quad$ Fig S2

461 A. Cryptoccocal cells were grown in the presence or absence of IFNa over 24 hours. Growth

462 was assessed by optical densitiy readings at $600 \mathrm{~nm}$.

463 B-C. Human monocyte-derived macrophages were infected with $C$. neoformans in the

464 presence of different doses of recombinant IFNa. Time-lapse microscopy videos were

465 manually scored for phagocytosis and intracellular proliferation rate of the fungus (B and C,

466 respectively).

467 Data corresponds to 3 independent experiments. 


\section{Measles infection}
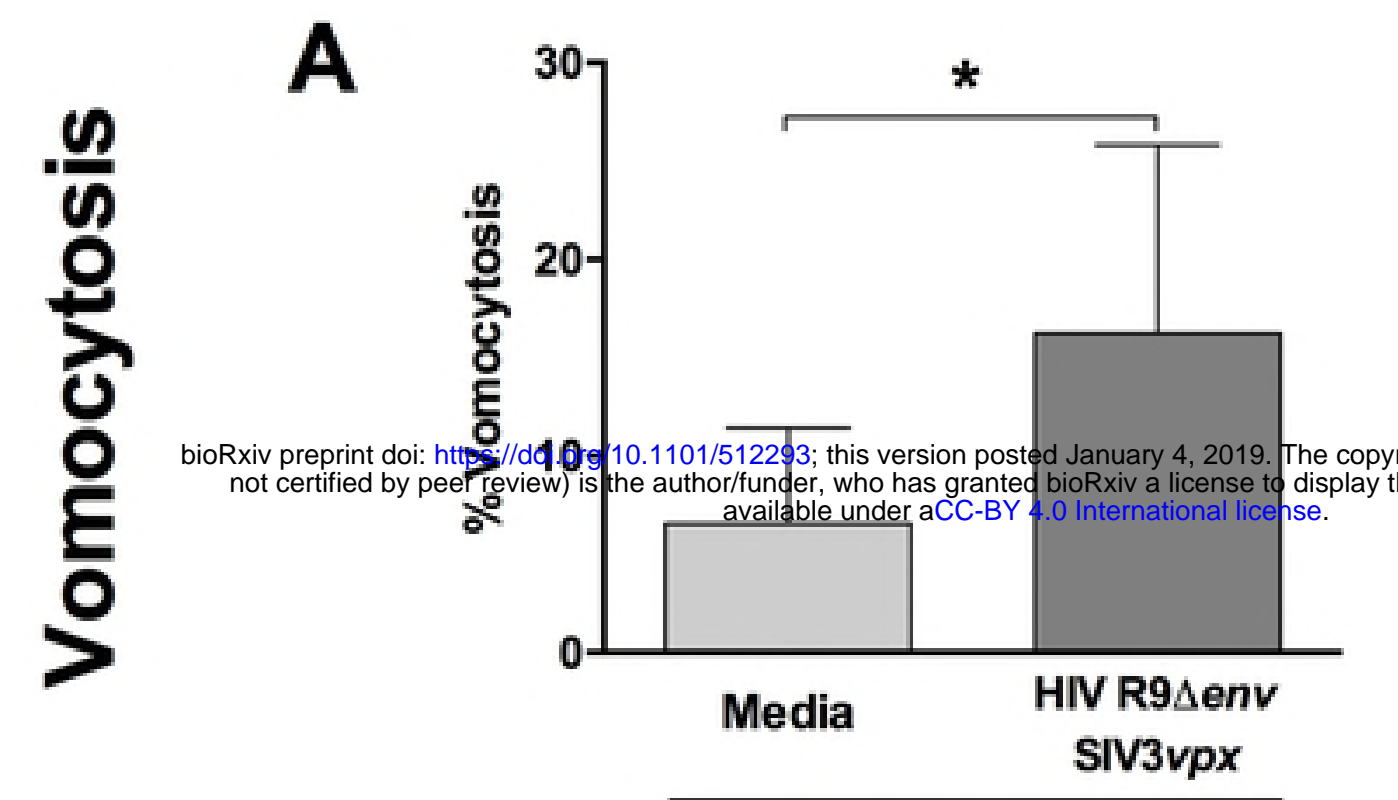

Kn99 $\alpha$-mCherry

B

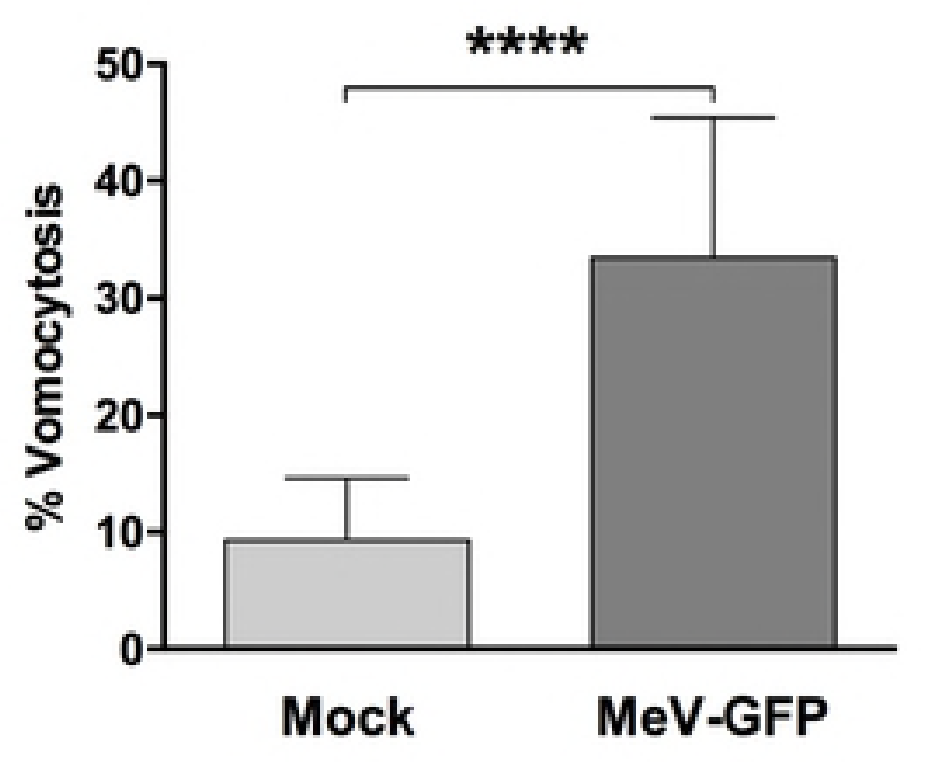

C

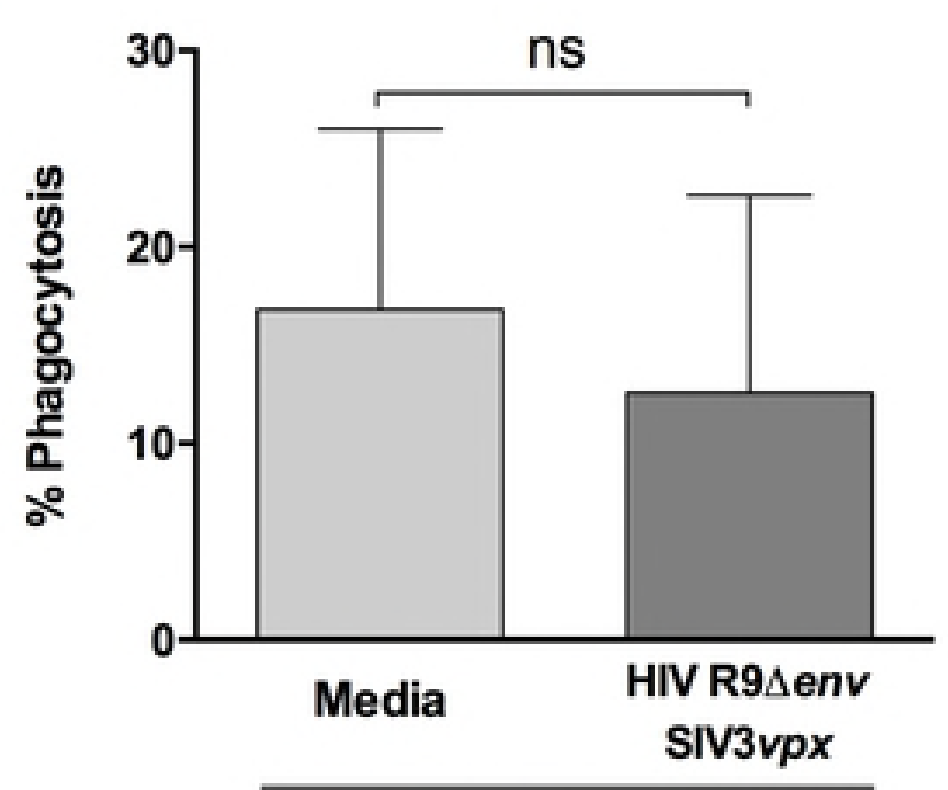

Kn99 $\alpha$-mCherry

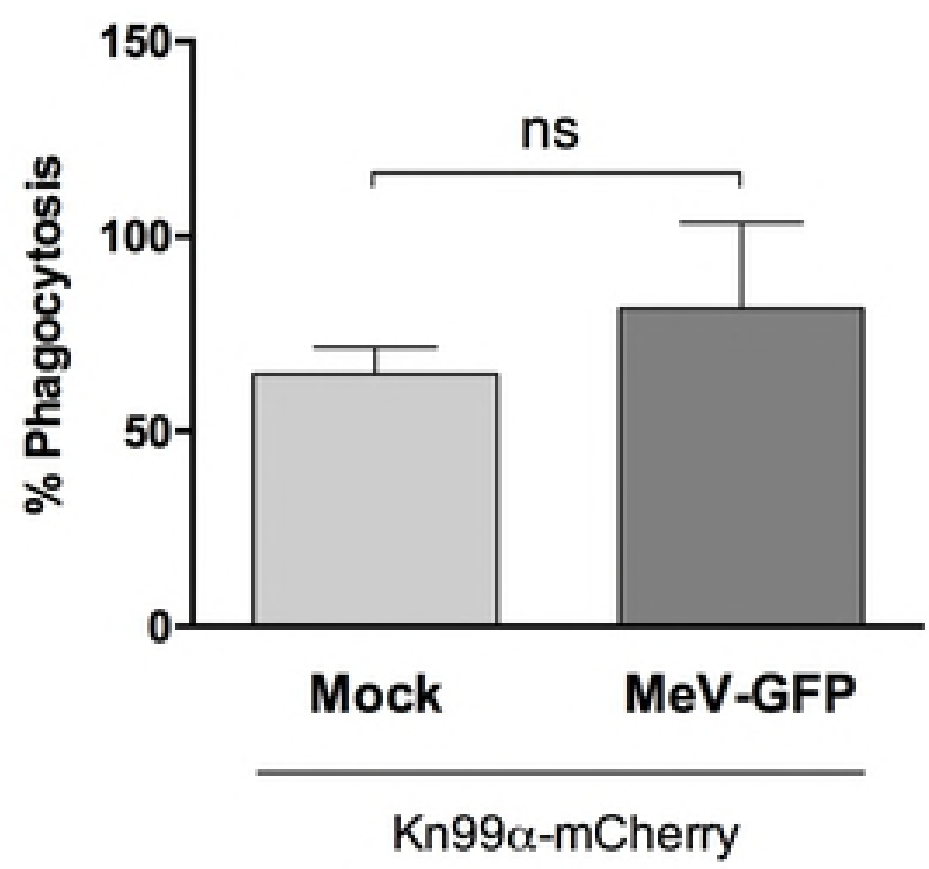

E
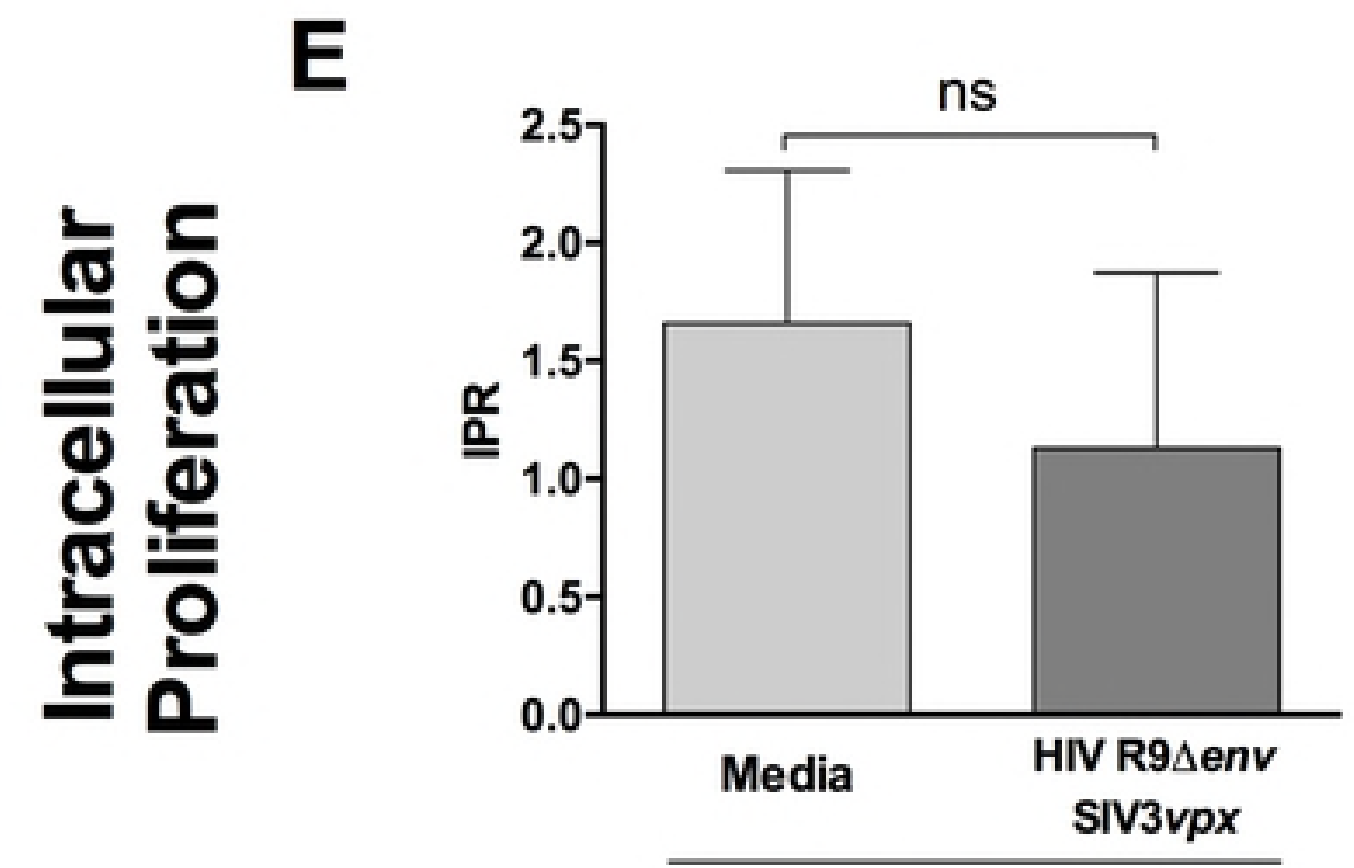

Kn99 $\alpha$-mCherry

D

Kn99 $\alpha$-mCherry

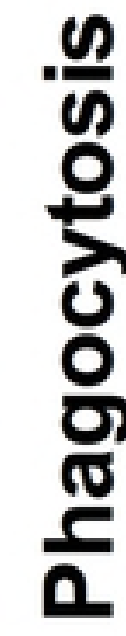

F

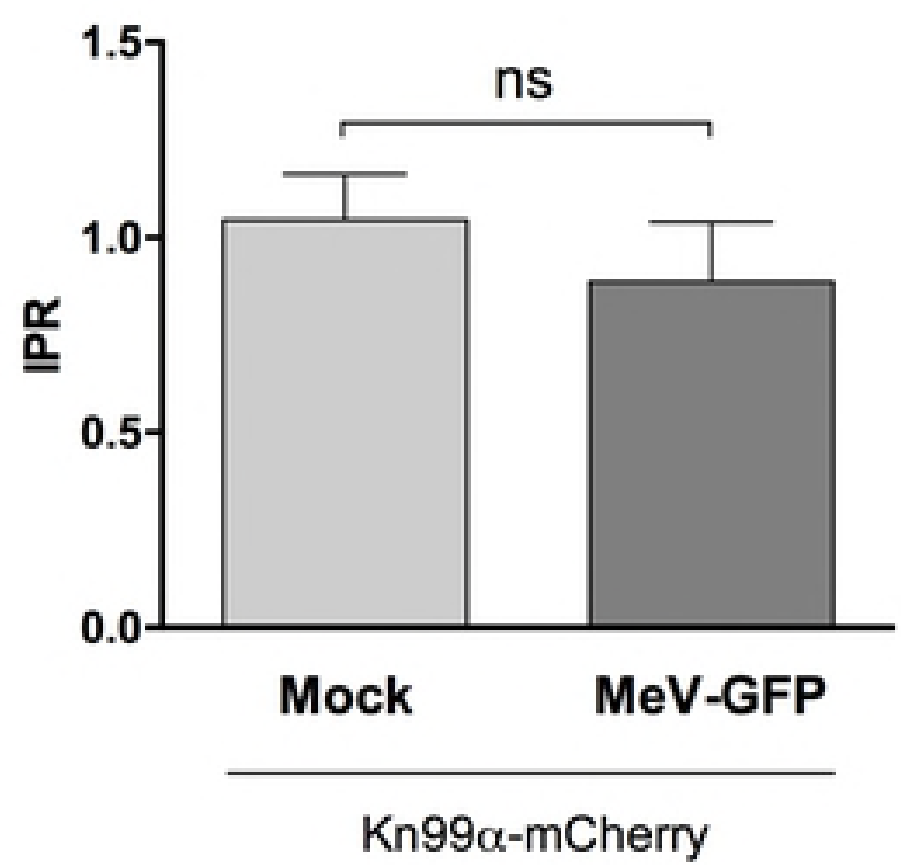

Figure 1 
A

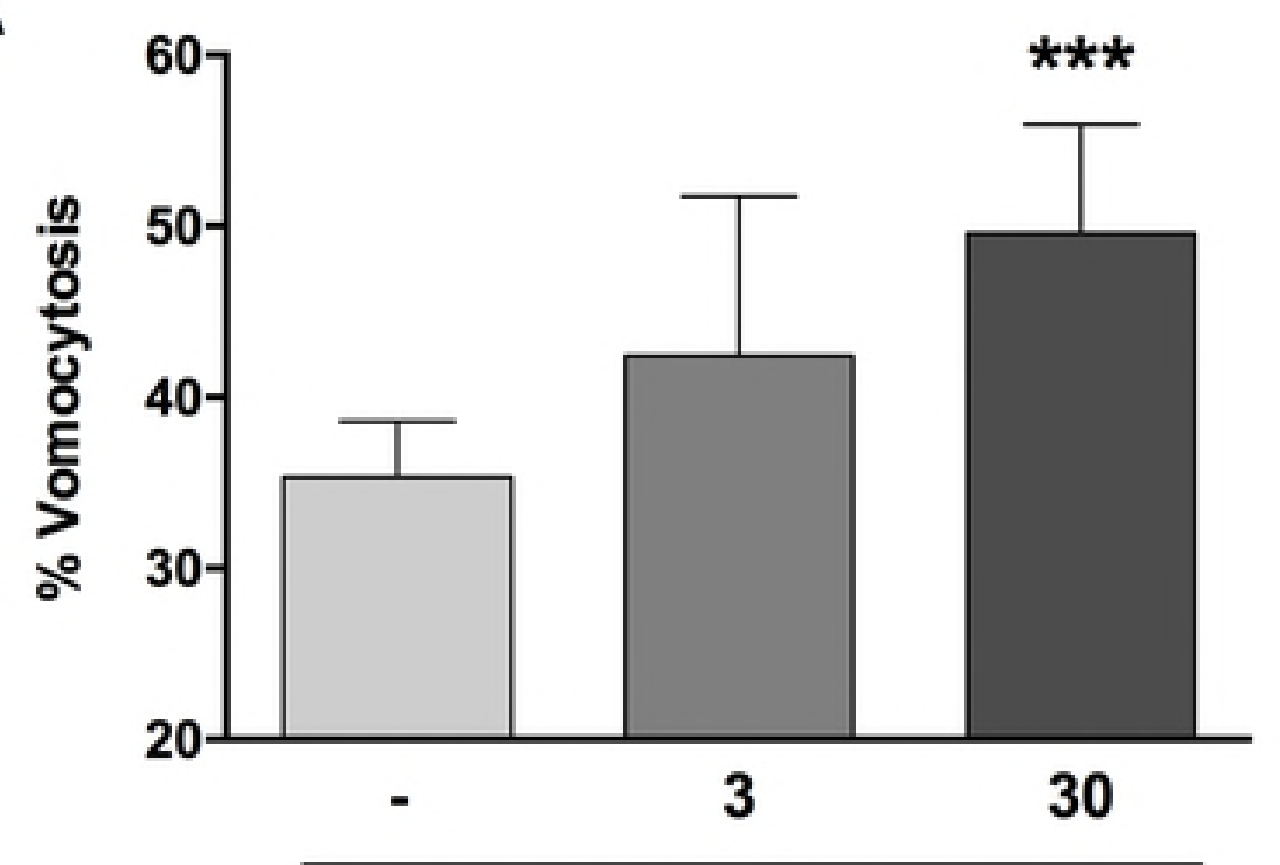

polylC (ng/mL)

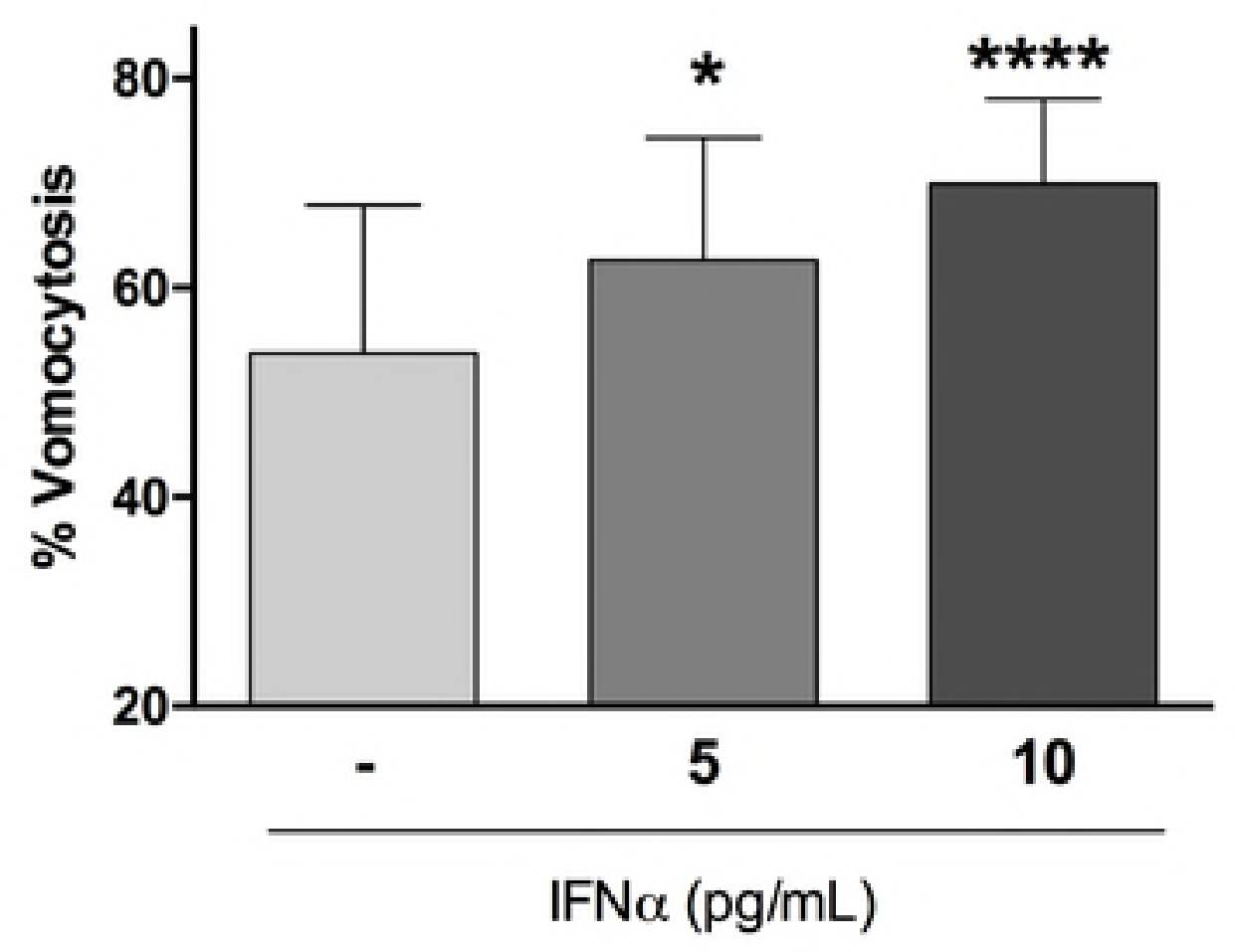

Figure 2 


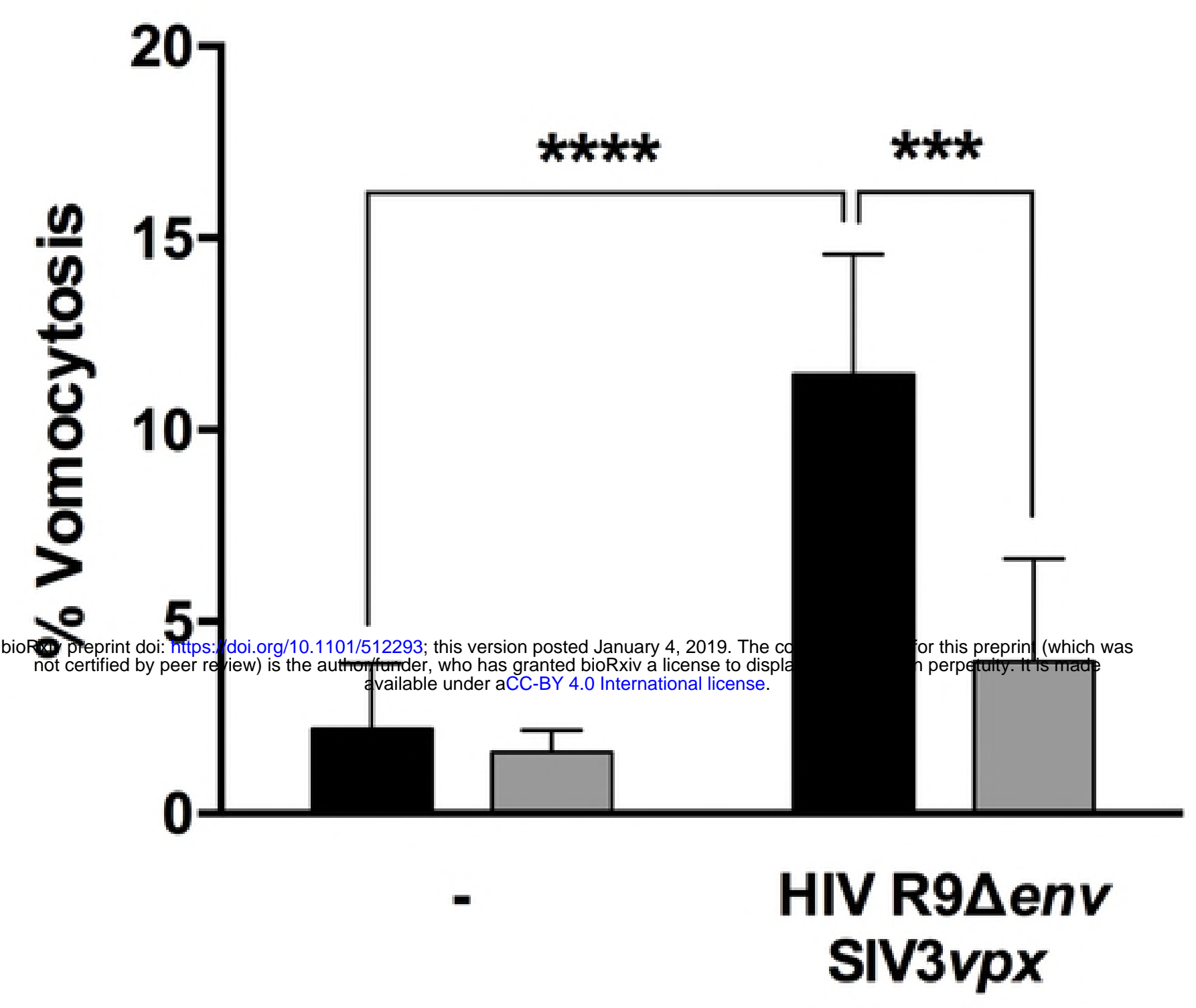

Kn99 $\alpha$-mCherry

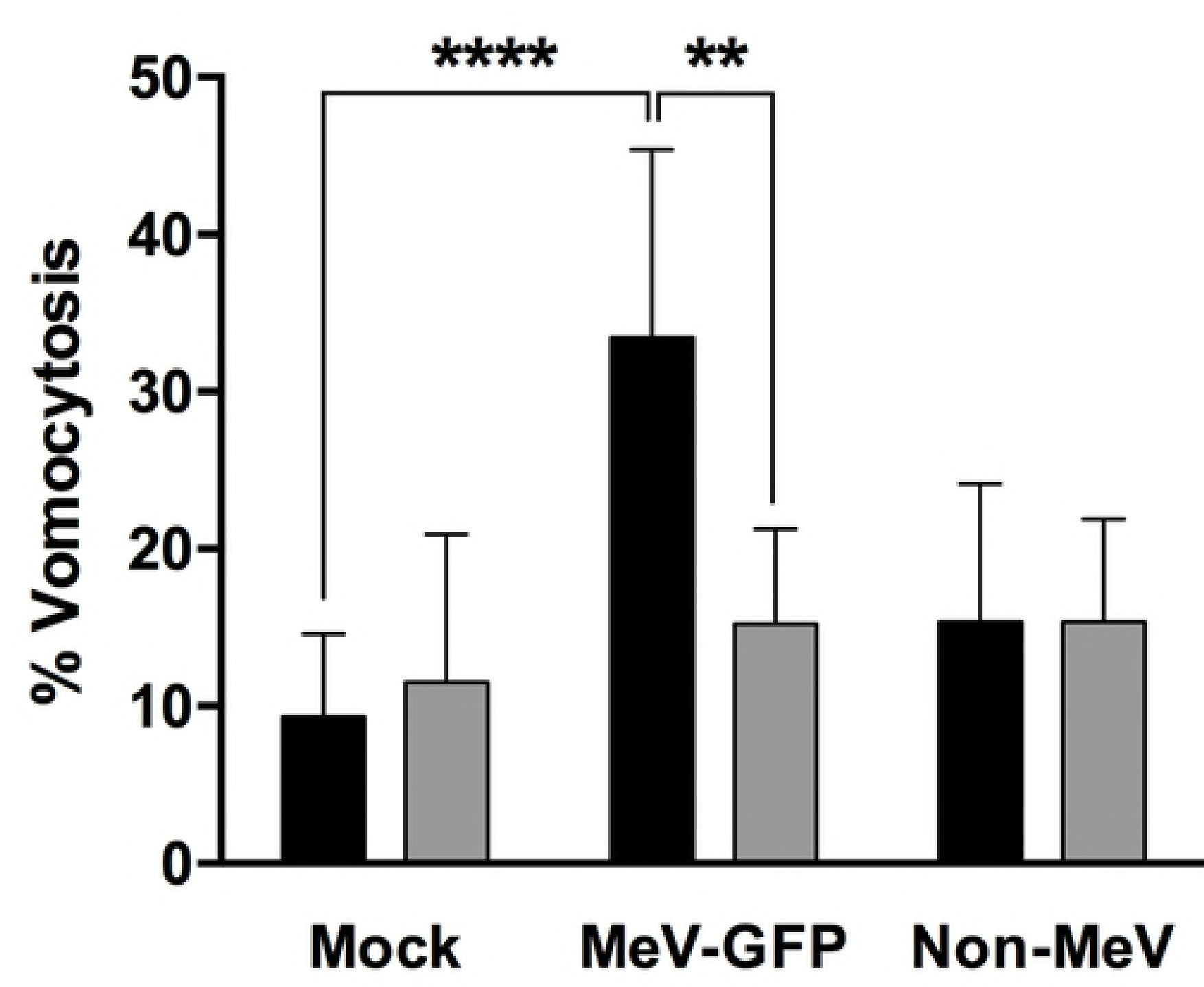

Kn99a-mCherry

Figure 3 